\title{
Semiconductor Quantum Dots Surface Modification for Potential Cancer Diagnostic and Therapeutic Applications
}

\author{
Jidong Wang, ${ }^{1}$ Shumin Han, ${ }^{1,2}$ Dandan Ke, ${ }^{1}$ and Ruibing Wang ${ }^{3}$ \\ ${ }^{1}$ College of Environmental and Chemical Engineering, Yanshan University, Qinhuangdao 066004, China \\ ${ }^{2}$ State Key Laboratory of Metastable Materials Science and Technology, Yanshan University, Qinhuangdao 066004, China \\ ${ }^{3}$ Targeted Therapy Unit, Nordion Inc., 447 March Road, Ottawa, ON, Canada K2K 1X8
}

Correspondence should be addressed to Shumin Han, hanshm@ysu.edu.cn and Ruibing Wang, ruibing.wang@nordion.com

Received 4 September 2012; Accepted 20 September 2012

Academic Editor: Haiyan Li

Copyright (c) 2012 Jidong Wang et al. This is an open access article distributed under the Creative Commons Attribution License, which permits unrestricted use, distribution, and reproduction in any medium, provided the original work is properly cited.

\begin{abstract}
Semiconductor Quantum dots (QDs) have generated extensive interest for biological and clinical applications. These applications arise from their unique properties, such as high brightness, long-term stability, simultaneous detection of multiple signals, tunable emission spectra. However, high-quality QDs, whether single or core-shell QDs, are most commonly synthesized in organic solution and surface-stabilized with hydrophobic organic ligands and thus lack intrinsic aqueous solubility. For biological applications, very often it is necessary to make the QDs dispersible in water and therefore to modify the QD surfaces with various bifunctional surface ligands or caps to promote solubility in aqueous media. Well-defined methods have been developed for QD surface modification to impart biocompatibility to these systems. In this review, we summarize the recent progress and strategies of QDs surface modification for potential cancer diagnostic and therapeutic applications. In addition, the question that arose from QD surface modification, such as impact of size increase of QD bioconjugates after surface-functionalization or surface modification on photophysical properties of QDs, are also discussed.
\end{abstract}

\section{Introduction}

Cancer is one of the most serious health threats worldwide, with an estimated 12.7 million new cases and 7.6 million cancer deaths in 2008 [1]. Cancer is greatly feared due to the widespread occurrence of this disease, its high death rate, and recurrence after treatment. The application of nanotechnology in medicine has provided an unprecedented opportunity for addressing many of the current challenges in the diagnosis and treatment of cancer $[2,3]$.

Semiconductor quantum dots (QDs) have great potential in biomedical fields, due to their excellent optical properties. QDs exhibit discrete size-dependent energy levels. As the size of the nanocrystal increases, the energy gap decreases, yielding a size-dependent rainbow of colors. By varying the size and the composition of the QDs, light wavelengths from the ultraviolet to the infrared range can be achieved, so that they enable simultaneous examination of multiple molecules and events [4]. QDs have unique optical spectra compared to organic fluorophores. Organic dyes typically have narrow absorption spectra, which mean that they can only be excited within a narrow window of wavelengths. Furthermore, organic dyes have asymmetric emission spectra broadened by a red tail. In contrast, QDs have broad absorption spectra, enabling excitation by a wide range of wavelengths, and their emission spectra are symmetric and narrow. Consequently, multicolor nanocrystals of different sizes can be excited by a single wavelength shorter than their emission wavelengths, with minimum signal overlap. QDs are also relatively stable light emitters owing to their inorganic composition, making them less susceptible to photobleaching than organic dye molecules $[5,6]$. The unique optical properties of QDs make them appealing as in vivo and in vitro fluorophores in various biological and medical applications, such as diagnosis and therapy of cancer [7-11].

QDs are usually utilized as either core-only or core-shell structures [12]. Core-shell nanoparticles are more desirable for biological applications as the core being passivated by the shell (usually a wider band-gap material), which improves fluorescent properties and prevents leaching. Core-shell 
QDs are typically synthesized from various semiconductor materials which can provide access to a full range of potential emission wavelengths. High-quality QDs, whether single or core-shell structures, are most commonly synthesized in organic solution and surface-stabilized with hydrophobic organic ligands and thus lack intrinsic aqueous solubility. For biological applications, it is necessary to alter the QDs so that they become dispersible in water, and therefore to modify the QDs surface with various bifunctional surface ligands or caps to promote solubility in aqueous media $[4,13-$ 16]. Also, the attachment of biological molecules to the QD surface needs to provide the desired specific biorecognition and/or targeting properties. In some cases, the QD surface ligands must accomplish this mission by providing unique functional groups, such as carboxyls or amines, for the multistep covalent chemical coupling of biomolecules. Therefore, surface modification is vital for QDs in biological applications. In this paper, we summarize the recent progress and strategies in QDs surface modification for cancer diagnostics and therapeutics related applications: (1) the growth of additional layer, for instance a silica shell, around the nanoparticles, through surface silanization; (2) the exchange of hydrophobic surfactant molecules with bifunctional molecules; (3) coating of QD surfaces with amphiphilic polymers, phospholipid micelles, or microspheres. Moreover, the questions that arose from QD surface modification, such as the impact size increase of QD bioconjugates after surface functionalization or surface modification on photophysical properties of QDs, are also discussed.

\section{Silanization}

In order to render QDs more effective and also more biocompatible for potential cancer diagnostics and therapeuticrelated biomedical applications, one of the most preferred approaches is to introduce a silica shell covering the QDs [17, 18]. This approach is also called surface silanization $[6,19]$. The first step of this process involves exchanging the surface ligand with a thiol-derived silane, such as mercaptopropyltris(methyloxy)silane (MPS). The trimethoxysilane groups can be cross-linked by the formation of siloxane bonds. During further silica shell growth, other types of silicon can be added to render a different charge and provide functional groups on the surface (Figure 1) [4]. Those most frequently used are aminopropylsilanes (APS), phosphosilanes, and polyethylene glycol (PEG)-silane $[19,20]$. Because the silica shells are highly cross-linked, silanized QDs are extremely stable. Silanization is preferred also because it is less toxic in comparison with other ligands $[21,22]$. Most reports of silica coated systems are limited up to the cytotoxicity evaluation [23-25]. For example, for in vivo applications, these materials have to travel through body fluids such as like blood. In that sense, the materials have to satisfy the mandatory criteria such as basic haemocompatibility [26].

A number of reports have been devoted to silica coating of colloidal nanoparticles by aqueous classical methods such as Stöber synthesis methodology [27-29], silicate water-glass methodology [30, 31], and the methodology using sodium of silane coupling agents [32]. Recently, many new approaches have been explored. Based on classical methods, GuerreroMartínez et al. [30] developed three different synthetic strategies, including: (i) the use of water-in-oil (W/O) microemulsions to coat nanoparticles such as metal colloids, magnetic nanoparticles, and semiconductor nanocrystals, (ii) silica coating of polymeric aggregates, surfactant vesicles, and polymer/surfactant stabilized inorganic nanoparticles, and (iii) assembly of silica colloids on nano- and microparticles by various physiosorption strategies (Figure 2). Although many groups have also successfully prepared silica-coated core-shell QDs, the various types of surface-silanized QDs still could be divided into three classes, such as single QDs, core-shell QDs, and multiple-layered QDs being coated by silica shell.

Firstly, Chen et al. [33] synthesized CdTe QDs coated with silica particles and the particles surface was further functionalized covalently with $\alpha$-fetoprotein antibody, antiAFP (secondary antibodies denoted $\mathrm{Ab}_{2}$ ). This work presents a novel strategy for ultrasensitive detection of biomarkers based on CdTe quantum dot. The enhanced detection of biomarkers based on single QD-functionalized silica nanosphere labels was achieved by an increase in CdTe QDs loading per sandwiched immunoreaction. Tan et al. [34] utilized silica functionalized PbSe QDs on two different cell lines for cytotoxic studies and found that the silica-coated PbSe QDs were much less cytotoxic than the polymer coated PbSe QDs, which were used for comparison.

More interestingly, Selvan et al. [35] employed Igepal as a nonionic surfactant and synthesized $20 \sim 30 \mathrm{~nm}$ silica-coated $\mathrm{CdSe} / \mathrm{ZnS} / \mathrm{SiO}_{2}$ QDs via a reverse microemulsion. The QDs displayed comparable quantum yields to $\mathrm{CdSe} / \mathrm{ZnS}$ QDs, but were much less toxic than organic-coated water-soluble QDs. Also, Bruchez et al. [6] extended the $\mathrm{CdSe} / \mathrm{CdS} / \mathrm{SiO}_{2}$ coreshell systems by adding a third layer of silica to labeled 3T3 mouse fibroblast cells. Furthermore, Durgadas et al. [26] reported $\mathrm{CuSe} / \mathrm{ZnS} / \mathrm{Silica} \mathrm{QDs}$, which were conjugated with an anticancer drug and labeled HepG2 and C6 glioma cancer cells. They also pointed out that the improvement of the core/shell/shell QD synthesis would expand the applications of QDs in disease site targeting and cellular labeling.

Finally, Yi et al. [36] and Selvan et al. [37] encapsulated QDs and magnetic nanoparticles (MPs) in a silica shell using a reverse microemulsion synthesis. The resulting MPs $(\gamma$ $\mathrm{Fe}_{2} \mathrm{O}_{3}$ )-QDs ( $\left.\mathrm{CdSe}\right) / \mathrm{SiO}_{2}$ nanocomposite particles present a unique combination of magnetic and optical properties. Their nonporous silica shell facilitated their surface modification for bioconjugation in various biomedical applications. For example, the synthesis of multifunctional nanoparticles, in which both QDs and magnetic particles were coated with silica, was used for live cell imaging of human liver cancer cells (HepG2), mouse fibroblast cells (NIH-3T3), and the protein detection.

In general, these silica-coated core-shell nanostructured architectures are fascinating hybrid materials that combine various types of cores with silica that possess superior stability and little toxicity. In addition, the careful control over the thickness of the silica shell can provide sensitive tuning of the response to light and magnetic information. Silica-coated 

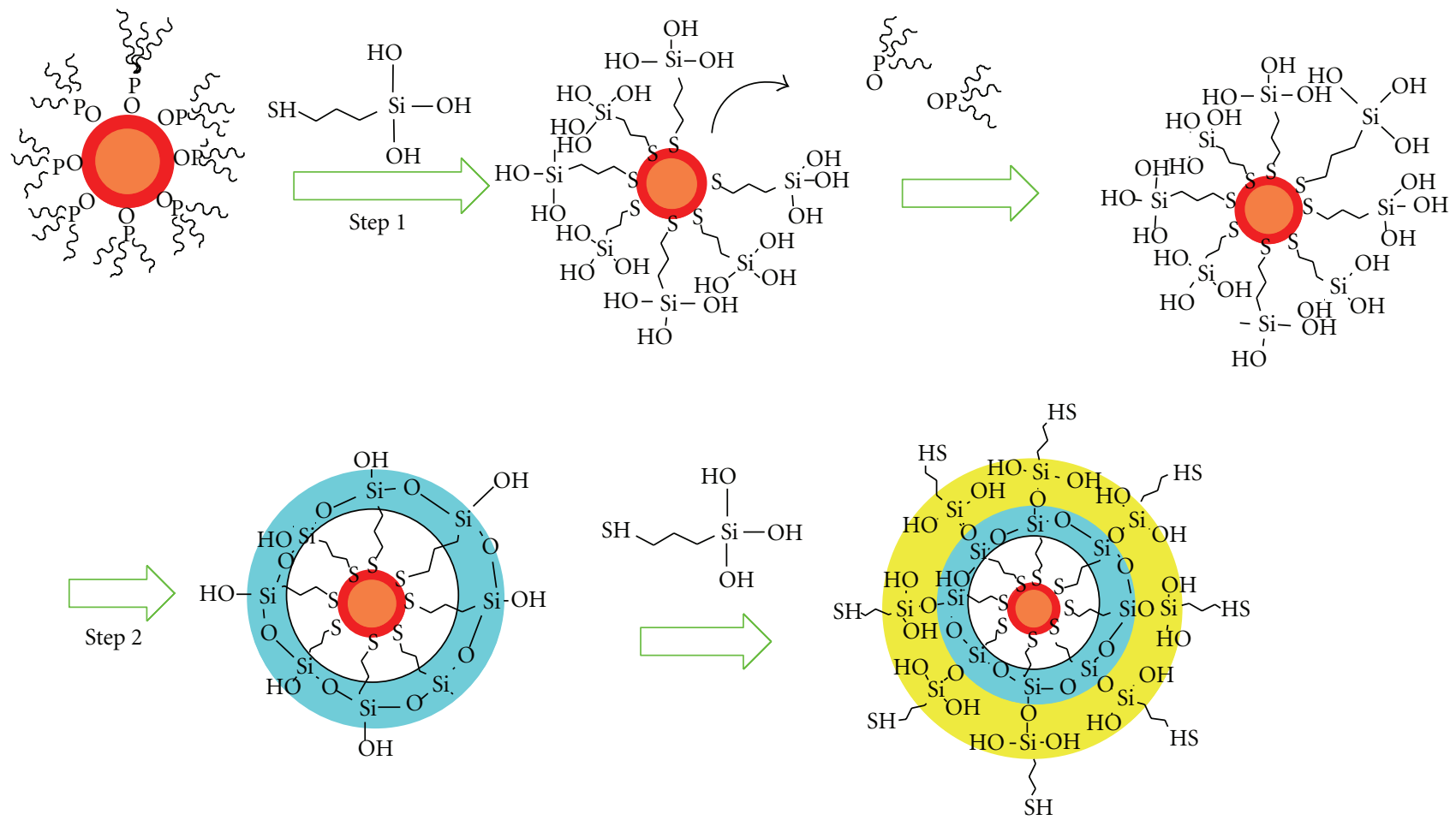

FIGURE 1: Surface silanization sketch of TOPO-capped CdSeZnS core/shell particles by mercaptopropyltris(methyloxy)silane (MPS). The diagram was adapted from [4].

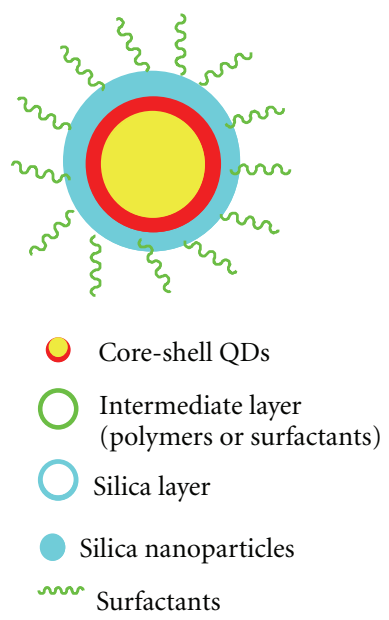

(a)

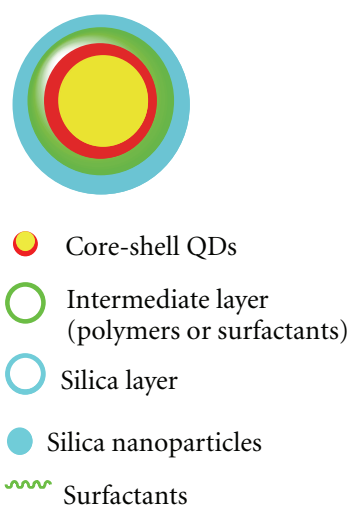

(b)
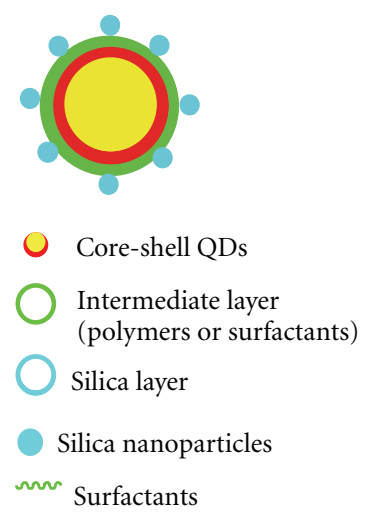

(c)

FIGURE 2: Three synthetic approaches of silica coated nanomaterials. (a) W/O microemulsion method, (b) silica coating the polymers or surfactants, (c) silica nanoparticles self-assembled onto nanomaterials.

systems also could be beneficial for electronic and biosensing applications.

\section{Ligand Exchange}

Among the strategies that have been used to stabilize core or core-shell nanocrystals in aqueous solutions, the most common approach is to exchange the hydrophobic surfactant molecules with bifunctional molecules, which are hydrophilic on one end and hydrophobic on the other end. Because these bifunctional molecules replace the hydrophobic surfactants, this process is called ligand exchange [19]. Among the bifunctional molecules, thiols $(-\mathrm{SH})$ are used as anchoring groups to bind to the QDs surface, while carboxyl $(-\mathrm{COOH})$ groups are used as the hydrophilic ends. Numerous examples of QD surface ligand exchange with using bifunctional biorelevant molecules such as cysteine, 
mercaptosuccinic acid, and glutathione have provided finished materials with opportunities for biomedical applications [38].

3.1. Cysteine. Liu et al. [39] developed a compact cysteine coating on a highly robust $\mathrm{CdSe} / \mathrm{ZnCdS} \mathrm{QD}$ to form watersoluble QDs via ligand exchange. These QDs were biologically compatible, extraordinarily compact, highly fluorescent, and easily functionalized nanocrystals for in vivo targeted imaging. Choi et al. [40] also coated CdSe/ZnS QDs with cysteine by ligand exchange and precisely defined the requirements for renal filtration and urinary excretion of inorganic, metal-containing nanoparticles. They reported that the hydrodynamic diameter (HD) was smaller than $5.5 \mathrm{~nm}$, which was beneficial for rapid and efficient urinary excretion and elimination of QDs from the body. This study provided a foundation for the design and development of biotargeting nanoparticles for biomedical applications [41, 42].

3.2. Mercaptosuccinic Acid. Yong et al. [43] functionalized the InP/ZnS QDs with mercaptosuccinic acid to render them highly dispersible in aqueous media and allowed specific in vitro targeting of pancreatic cancer cell lines. This work has suggested the immense potential of InP/ZnS QDs as safe and efficient non-cadmium-based optical imaging nanoprobes for the early detection of cancer.

3.3. Polyethylene Glycol. Choi et al. [44] coated InAs/ZnS QDs with various chain length of polyethylene glycol (PEG). They found that increasing the PEG chain length from 2 to 22 units not only resulted in changes in hydradynamic diameter, but also resulted in better targeting to the liver, kidney, pancreas, and lymph nodes.

3.4. Mercaptoacetic Acid. Yu et al. [45] synthesized mercaptoacetic acid (MAA) coated CdSe/ZnS QDs via ligand exchange and linked QDs to alphafetoprotein (AFP) antibodies for specific binding with AFP. They archived specific fluorescence detection with the QD-anti-AFP in nude mice xenocrafted with cancer. The fluorescence results demonstrated that QD-Anti-AFP fluorescent spectral and lifetimes had not varied much from that of the original QDs. Moreover, QD-Anti-AFP had exhibited higher fluorescence efficiencies than QDs under two-photon excitation.

3.5. Glutathione. Tiwari et al. [46] used glutathione to coat CdSe/CdZnS QDs (GSH-QDs) and anti-HER2 antibodies to conjugate QDs (HER2Ab-QDs) using different coupling agents (EDC(1-ethyl-3-(3-dimethyl-aminopropyl)carbodiimide)/sulfo-NHS(3-sulfo-N-hydroxy-succinimide sodium salt), iminothiolane/sulfo-SMCC(sulfosuccinimidyl-trans4-(N-maleimidomethyl)cyclohexane-1-carboxylate), and sulfo-SMCC). These fluorescent probes have great potential for the precise determination of HER2 status among breast cancer patients at the cellular levels.

3.6. Ligand Exchange with Other Molecules. QDs functionalized with polyamidoamine (PAMAM) [47-49] and polyisoprene [38] have shown great potential for applications in cancer targeting and molecular imaging, such as gastric cancer cells. The resulting water-soluble QDs have shown fluorescence quantum efficiencies in the 40 to $50 \%$ range and extraordinary fluorescence stability in biological environments after cross-linking of the ligand shell.

Although many biological applications of QDs have been achieved by using bifunctional molecules to render QDs water soluble, this approach can often negatively alter the chemical and physical states of the QDs surface and cause a dramatic decrease in the quantum efficiency. For example, Jin et al. [50] modified the surfaces of hydrophobic CdSeTe/ CdS QDs with GSH in a tetrahydrofuran-water solution and these QDs exhibited quantum yields of only 22\%. Moreover, the stability of the QDs has not been well understood under in vivo conditions. The long-term stability of the QDs depends on the bond between the thiol group and the QD surface, which can be weak with oxidation. Consequently, the thiol-based molecules can become cleaved from the QD surface, causing the QDs to aggregate and precipitate from solution.

\section{Amphiphilic Polymer Coatings}

Instead of exchanging the hydrophobic surfactant, the QDs in this case are coated with a cross-linked amphiphilic polymer. The hydrophobic part of the amphiphile is designed to interact with the hydrophobic chains of the surface ligands, and the hydrophilic component (most commonly in the form of carboxyl groups or polyethylene glycol chains) provides water solubility and chemical functionality.

The CdSe/ZnS QDs [58, 59], after being coated by PEG(poly(ethylene glycol)), were conjugated with monoclonal anti-EGFR (epidermal growth factor receptor) antibodies (cetuximab) through two long-chain heterobifunctional linkers, sulfo-LC-SPDP(sulfosuccinimidyl 6[3'-(2-pyridyldithio)propionamido]hexanoate) and sulfoSMCC(sulfosuccinimidyl-trans-4-(N-maleimidomethyl)cyclohexane-1-carboxylate). The cellular imaging experiments using the QD-cetuximab conjugates showed a clear endocytosis pathway, which was evidenced by the colocalization of the QD-cetuximab conjugates with dye-labeled transferrin. These results suggest that the QD-cetuximab conjugates as an imaging modality for tumor EGFR overexpression can be expected to provide important information on the expression levels of EGFR among cancer cells. Such applications include in vitro assays, cellular imaging, and in vivo imaging applications.

During amphiphilic polymerization coating process, a cross-linking treatment is often performed. Although not always necessary, the cross-linking reactions are thought to increase the stability of the polymeric shell of the QDs. Hydrolysis of the remaining anhydride groups transfers the polymer-coated QDs to the water phase. This treatment also provides a large number of carboxylic units, which can be used for further derivatization. Although there are relatively few literature examples of QDs coated with amphiphilic polymers, the methods based on hydrophobic interactions between the QD ligands and polymer functionalities have 
TABLE 1: QD/microbead hybrid material preparation methods and their characteristics and biomedical applications.

\begin{tabular}{|c|c|c|}
\hline Approaches & QDs & Characteristics or applications \\
\hline \multirow{4}{*}{$\begin{array}{l}\text { QDs are absorbed into the subsurface } \\
\text { region of microbeads, as the solvent is } \\
\text { removed }\end{array}$} & \multirow{3}{*}{ (a) CdSe/ZnS [51] } & (1) Bead identification accuracies as high as $99.99 \%$ \\
\hline & & (2) The coding and target signals of DNA hybridization at the \\
\hline & & $\begin{array}{l}\text { single-bead level } \\
\text { (1) Both single- and dual-color codes were also obtained }\end{array}$ \\
\hline & (b) $\mathrm{CdSeTe}[52]$ & $\begin{array}{l}\text { (2) Good detection sensitivity and low cytotoxicity on suspension } \\
\text { immunoassay for goat antimouse IgG on the xMAP platform }\end{array}$ \\
\hline \multirow{2}{*}{$\begin{array}{l}\text { QDs are electrostatically bound to the } \\
\text { surfaces of the microspheres, using } \\
\text { layer-by-layer strategy }\end{array}$} & (a) $\mathrm{CdTe}[53]$ & $\begin{array}{l}\text { More flexibility for creating QD-beads in biomedicine applications } \\
\text { (sensing, immunoassay, encoding, and diagnostic) }\end{array}$ \\
\hline & (b) $\mathrm{CdTe}[54]$ & Cytotoxicity reductions \\
\hline $\begin{array}{l}\text { QDs grow around preformed silica } \\
\text { spheres }\end{array}$ & $\begin{array}{l}\text { CdSe, CdSe/ZnS, or } \\
\text { CdSe/ZnSe/ZnS [55] }\end{array}$ & A comparatively stable and noncytotoxic intracellular delivery \\
\hline \multirow{2}{*}{$\begin{array}{l}\text { QDs are incorporated into polymer } \\
\text { microbeads through emulsion and } \\
\text { suspension polymerization }\end{array}$} & (a) CdSe/ZnS [56] & $\begin{array}{l}\text { Good detection sensitivity of rabbit IgG molecules onto the surfaces of } \\
\text { the microbeads-QDs }\end{array}$ \\
\hline & (b) $\mathrm{Cd}_{1-x} \mathrm{Zn}_{x} \mathrm{Se}_{1-y} \mathrm{~S}_{y}[57]$ & $\begin{array}{l}\text { High-throughput multiplexed biomolecular immunoassay for human } \\
\text { IgG detection }\end{array}$ \\
\hline
\end{tabular}

already addressed many problems related to undesired ligand exchange reactions.

\section{Micellar Phospholipid Coatings}

The encapsulation of QDs within phospholipid micelles is attractive for several reasons [60-62]: firstly, the encapsulation step does not alter the surface, secondly, the optical properties of the QDs are retained, and thirdly, the micelles have highly dense surfaces, which prevents nonspecific adsorption so that the supramolecular architecture is maintained by local hydrophobic interactions.

Dubertret et al. [63] encapsulated individual CdSe/ZnS QDs in phospholipid block copolymer micelles and demonstrated both in vitro and in vivo imaging. When conjugated to DNA, the nanocrystal-micelles acted as in vitro fluorescent probes to hybridize to specific complementary sequences. In comparison with other systems, they simultaneously provided efficient fluorescence, a great reduction in photobleaching, colloidal stability in various biological environments, and low nonspecific adsorption. Hu et al. [64] also reported alloyed $\mathrm{CdTe}_{1-x} \mathrm{Se}_{x} / \mathrm{CdS}$ QDs encapsulated within PEG-grafted phospholipid micelles. For tumorspecific delivery in vivo, the micelle-encapsulated QDs were conjugated with a cyclic arginine-glycine-aspartic acid (cRGD) peptide, which targeted the $\alpha_{v} \beta_{3}$ integrins that are overexpressed in angiogenic tumor vasculatures. Their work had demonstrated that these QDs provide a promising theranostic, nanosized platform for both cancer imaging and therapy. Erogbogbo et al. [65] reported that silicon QDs and iron oxide nanoparticles were coencapsulated by phospholipid-polyethylene glycol (DSPE-PEG) micelles. Their luminescence stability in a prostate cancer microenvironment was demonstrated in vivo. Many groups also have synthesized lipid-coated nanoparticles and used them in cellular and in vivo imaging [66-70]. The encapsulation of QDs by phospholipid micelles provides more opportunities for utilizing QDs in biological applications.

\section{Microsphere/Microbead Coatings}

Incorporating QDs in microspheres is of interest for both fundamental studies on light-matter interactions [71] and practical applications, such as semiconductor microlasing [72] and biological tags [6]. This dot-in-a-dot structure confines electrons and photons in all three dimensions. The spontaneous emission was enhanced from that of regular QDs because the QDs were embedded within the microcavities [73].

Methods to incorporate QDs into microbeads include the following approaches [74]. The first approach involves the dispersion of commercially available or synthesized microbeads and QDs in a solvent/nonsolvent mixture is chosen to cause the partial swelling of microbeads. QDs that are dispersed in the solution penetrate into the microbeads and, as the solvent is removed, are captured in the subsurface region of the microbeads. The second method utilizes a layer-by-layer strategy to adsorb QDs consecutively onto oppositely charged microbeads. In this scheme, QDs are electrostatically bound to the surfaces of the microspheres. The third approach involves the growth of a silica/QD shell around preformed silica spheres. Finally, the fourth approach involves loading QDs into polymer microbeads through emulsion and suspension polymerization. With these methods described above, various QDs can be incorporated into microspheres/microbeads and are further directed to more biomedical applications such as diagnostics (Table 1).

\section{Summary and Future Challenges}

The rapid development of nanotechnology has given the new hope in the early detection and potentially treatment of cancer. Much basic work on the engineering of QD surfaces and creating QD bioconjugates with control over all relevant properties has been accomplished. In spite of this, there are still many questions that remain unanswered. For example, size of a conjugate is also one crucial aspect. Although the sizes of QDs are comparable to those of 
large proteins, it is important to note that the surfacefunctionalization and further conjugation of biomolecules can increase the sizes of QD bioconjugates. Such large sizes may limit access to intracellular organelles and alter the intrinsic activity of any attached proteins. Therefore, developing surface functionalization techniques that maintain a small overall size is highly desirable. Additionally, during exchange between hydrophobic surfactant molecules and bifunctional molecules, the long-term stability of the QDs become more dependent on thiol- and metal bonds, which are relatively weak. The real-world applications of thiolcapped core-shell QDs are therefore rather limited. All of these considerations would hinder the application of QDs in biomedical applications. Therefore, the development of an effective approach for QD surface functionalization still remains a challenge for this technology.

\section{References}

[1] A. Jemal, F. Bray, M. M. Center, J. Ferlay, E. Ward, and D. Forman, "Global cancer statistics," CA Cancer Journal for Clinicians, vol. 61, no. 2, pp. 69-90, 2011.

[2] S. M. Hanash, C. S. Baik, and O. Kallioniemi, "Emerging molecular biomarkers-blood-based strategies to detect and monitor cancer," Nature Reviews Clinical Oncology, vol. 8, no. 3, pp. 142-150, 2011.

[3] J. A. Ludwig and J. N. Weinstein, "Biomarkers in cancer staging, prognosis and treatment selection," Nature Reviews Cancer, vol. 5, no. 11, pp. 845-856, 2005.

[4] A. P. Alivisatos, W. Gu, and C. Larabell, "Quantum dots as cellular probes," Annual Review of Biomedical Engineering, vol. 7, pp. 55-76, 2005.

[5] X. Michalet, F. Pinaud, T. D. Lacoste et al., "Properties of fluorescent semiconductor nanocrystals and their applications to biological labeling," Single Molecules, vol. 2, no. 4, pp. 261276, 2001.

[6] M. Bruchez, M. Moronne, P. Gin, S. Weiss, and A. P. Alivisatos, "Semiconductor nanocrystals as fluorescent biological labels," Science, vol. 281, no. 5385, pp. 2013-2016, 1998.

[7] A. P. Alivisatos, "Semiconductor clusters, nanocrystals, and quantum dots," Science, vol. 271, no. 5251, pp. 933-937, 1996.

[8] W. C. W. Chan and S. Nie, "Quantum dot bioconjugates for ultrasensitive nonisotopic detection," Science, vol. 281, no. 5385, pp. 2016-2018, 1998.

[9] P. Mitchell, "Turning the spotlight on cellular imaging," Nature Biotechnology, vol. 19, no. 11, pp. 1013-1017, 2001.

[10] W. C. W. Chan, D. J. Maxwell, X. Gao, R. E. Bailey, M. Han, and S. Nie, "Luminescent quantum dots for multiplexed biological detection and imaging," Current Opinion in Biotechnology, vol. 13, no. 1, pp. 40-46, 2002.

[11] X. Michalet, F. F. Pinaud, L. A. Bentolila et al., "Quantum dots for live cells, in vivo imaging, and diagnostics," Science, vol. 307, no. 5709, pp. 538-544, 2005.

[12] J. B. Delehanty, H. Mattoussi, and I. L. Medintz, "Delivering quantum dots into cells: strategies, progress and remaining issues," Analytical and Bioanalytical Chemistry, vol. 393, no. 4, pp. 1091-1105, 2009.

[13] I. L. Medintz, H. T. Uyeda, E. R. Goldman, and H. Mattoussi, "Quantum dot bioconjugates for imaging, labelling and sensing," Nature Materials, vol. 4, no. 6, pp. 435-446, 2005.

[14] F. Dubois, B. Mahler, B. Dubertret, E. Doris, and C. Mioskowski, "A versatile strategy for quantum dot ligand exchange,"
Journal of the American Chemical Society, vol. 129, no. 3, pp. 482-483, 2007.

[15] P. Alivisatos, "The use of nanocrystals in biological detection," Nature Biotechnology, vol. 22, no. 1, pp. 47-52, 2004.

[16] J. M. Klostranec and W. C. W. Chan, "Quantum dots in biological and biomedical research: recent progress and present challenges," Advanced Materials, vol. 18, no. 15, pp. 19531964, 2006.

[17] P. Mulvaney, L. M. Liz-Marzán, M. Giersig, and T. Ung, "Silica encapsulation of quantum dots and metal clusters," Journal of Materials Chemistry, vol. 10, no. 6, pp. 1259-1270, 2000.

[18] Y. Yang and M. Gao, "Preparation of fluorescent $\mathrm{SiO}_{2}$ particles with single CdTe nanocrystal cores by the reverse microemulsion method," Advanced Materials, vol. 17, no. 19, pp. 2354-2357, 2005.

[19] D. Gerion, F. Pinaud, S. C. Williams et al., "Synthesis and properties of biocompatible water-soluble silica-coated CdSe/ZnS semiconductor quantum dots," Journal of Physical Chemistry B, vol. 105, no. 37, pp. 8861-8871, 2001.

[20] W. J. Parak, D. Gerion, T. Pellegrino et al., "Biological applications of colloidal nanocrystals," Nanotechnology, vol. 14, no. 7, pp. R15-R27, 2003.

[21] F. Tamanoi, J. Lu, M. Liong, Z. Li, and J. I. Zink, "Biocompatibility, biodistribution, and drug-delivery efficiency of mesoporous silica nanoparticles for cancer therapy in animals," Small, vol. 6, no. 16, pp. 1794-1805, 2010.

[22] C. Chang, S. H. Wu, Y. S. Lin et al., "Multifunctional mesoporous silica nanoparticles for intracellular labeling and animal magnetic resonance imaging studies," ChemBioChem, vol. 9, no. 1, pp. 53-57, 2008.

[23] D. Wang, J. Qian, F. Cai, S. He, S. Han, and Y. Mu, "'Green'synthesized near-infrared $\mathrm{PbS}$ quantum dots with silicaPEG dual-layer coating: ultrastable and biocompatible optical probes for in vivo animal imaging," Nanotechnology, vol. 23, no. 24, pp. 245701-245702, 2012.

[24] P. Pericleous, M. Gazouli, A. Lyberopoulou, S. Rizos, N. Nikiteas, and E. P. Efstathopoulos, "Quantum dots hold promise for early cancer imaging and detection," International Journal of Cancer, vol. 131, no. 3, pp. 519-528, 2012.

[25] D. Richards and A. Ivanisevic, "Inorganic material coatings and their effect on cytotoxicity," Chemical Society Reviews, vol. 41, no. 6, pp. 2052-2060, 2012.

[26] C. V. Durgadas, K. Sreenivasan, and C. P. Sharma, "Bright blue emitting $\mathrm{CuSe} / \mathrm{ZnS} /$ silica core/shell/shell quantum dots and their biocompatibility," Biomaterials, vol. 33, no. 27, pp. 6420-6429, 2012.

[27] S. T. Selvanb, "Silica-coated quantum dots and magnetic nanoparticles for bioimaging applications (Mini-Review)," Biointerphases, vol. 5, no. 3, pp. FA110-FA115, 2010.

[28] M. Ohmori and E. Matijević, "Preparation and properties of uniform coated inorganic colloidal particles. 8. Silica on iron," Journal of Colloid And Interface Science, vol. 160, no. 2, pp. 288-292, 1993.

[29] L. M. Liz-Marzán and A. P. Philipse, "Synthesis and optical properties of gold-labeled silica particles," Journal of Colloid And Interface Science, vol. 176, no. 2, pp. 459-466, 1995.

[30] A. Guerrero-Martínez, J. Pérez-Juste, and L. M. Liz-Marzán, "Recent progress on silica coating of nanoparticles and related nanomaterials," Advanced Materials, vol. 22, no. 11, pp. 11821195, 2010.

[31] L. M. Liz-Marzán, M. Giersig, and P. Mulvaney, "Homogeneous silica coating of vitreophobic colloids," Chemical Communications, no. 6, pp. 731-732, 1996. 
[32] L. M. Liz-Marzán, M. Giersig, and P. Mulvaney, "Synthesis of nanosized gold-silica core-shell particles," Langmuir, vol. 12, no. 18 , pp. 4329-4335, 1996.

[33] L. Chen, C. Chen, R. Li, Y. Li, and S. Liu, "CdTe quantum dot functionalized silica nanosphere labels for ultrasensitive detection of biomarker," Chemical Communications, no. 19, pp. 2670-2672, 2009.

[34] T. T. Tan, S. T. Selvan, L. Zhao, S. Gao, and J. Y. Ying, "Size control, shape evolution, and silica coating of near-infraredemitting PbSe quantum dots," Chemistry of Materials, vol. 19, no. 13, pp. 3112-3117, 2007.

[35] S. T. Selvan, T. T. Tan, and J. Y. Ying, "Robust, non-cytotoxic, silica-coated CdSe quantum dots with efficient photoluminescence," Advanced Materials, vol. 17, no. 13, pp. 1620-1625, 2005.

[36] D. K. Yi, S. T. Selvan, S. S. Lee, G. C. Papaefthymiou, D. Kundaliya, and J. Y. Ying, "Silica-coated nanocomposites of magnetic nanoparticles and quantum dots," Journal of the American Chemical Society, vol. 127, no. 14, pp. 4990-4991, 2005.

[37] S. T. Selvan, T. T. Y. Tan, D. K. Yi, and N. R. Jana, "Functional and multifunctional nanoparticles for bioimaging and biosensing," Langmuir, vol. 26, no. 14, pp. 11631-11641, 2010.

[38] E. Pöselt, C. Schmidtke, S. Fischer et al., "Tailor-made quantum dot and iron oxide based contrast agents for in vitro and in vivo tumor," ACS Nano, vol. 6, no. 4, pp. 3346-3353, 2012.

[39] W. Liu, H. S. Choi, J. P. Zimmer, E. Tanaka, J. V. Frangioni, and M. Bawendi, "Compact cysteine-coated CdSe(ZnCdS) quantum dots for in vivo applications," Journal of the American Chemical Society, vol. 129, no. 47, pp. 14530-14531, 2007.

[40] H. S. Choi, W. Liu, P. Misra et al., "Renal clearance of quantum dots," Nature Biotechnology, vol. 25, no. 10, pp. 1165-1170, 2007.

[41] B. Tian, W. T. Al-Jamal, J. V. Bossche, and K. Kostarelos, "Design and engineering of multifunctional quantum dotbased nanoparticles for simultaneous therapeutic-diagnostic applications," Nanostructure Science and Technology, part 4, pp. 345-365, 2012.

[42] S. B. Lowe, J. A. G. . Dick, B. E. Cohen, and M. M. Stevens, "Multiplex sensing of protease and kinase enzyme activity via orthogonal coupling of quantum dot-peptide conjugates," ACS Nano, vol. 6, no. 1, pp. 851-857, 2012.

[43] K. T. Yong, H. Ding, I. Roy et al., "Imaging pancreatic cancer using bioconjugated inp quantum dots," ACS Nano, vol. 3, no. 3, pp. 502-510, 2009.

[44] H. S. Choi, B. I. Ipe, P. Misra, J. H. Lee, M. G. Bawendi, and J. V. Frangioni, "Tissue- and organ-selective biodistribution of NIR fluorescent quantum dots," Nano Letters, vol. 9, no. 6, pp. 2354-2359, 2009.

[45] X. Yu, L. Chen, Y. Deng et al., "Fluorescence analysis with quantum dot probes for hepatoma under one- and two-photon excitation," Journal of Fluorescence, vol. 17, no. 2, pp. 243-247, 2007.

[46] D. K. Tiwari, S. I. Tanaka, Y. Inouye, K. Yoshizawa, T. M. Watanabe, and T. Jin, "Synthesis and characterization of antiHER2 antibody conjugated CdSe/CdZnS quantum dots for fluorescence imaging of breast cancer cells," Sensors, vol. 9, no. 11, pp. 9332-9354, 2009.

[47] Z. Li, P. Huang, R. He et al., "Aptamer-conjugated dendrimermodified quantum dots for cancer cell targeting and imaging," Materials Letters, vol. 64, no. 3, pp. 375-378, 2010.

[48] D. A. Geraldo, E. F. Duran-Lara, D. Aguayo et al., "Supramolecular complexes of quantum dots and a polyamidoamine (PAMAM)-folate derivative for molecular imaging of cancer cells," Analytical and Bioanalytical Chemistry, vol. 400, no. 2, pp. 483-492, 2011.

[49] M. Akin, R. Bongartz, J. G. Walter et al., "PAMAM-functionalized water soluble quantum dots for cancer cell targeting," Journal of Materials Chemistry, vol. 22, no. 23, pp. 11529 11536, 2012.

[50] T. Jin, F. Fujii, Y. Komai, J. Seki, A. Seiyama, and Y. Yoshioka, "Preparation and characterization of highly fluorescent, glutathione-coated near infrared quantum dots for in vivo fluorescence imaging," International Journal of Molecular Sciences, vol. 9, no. 10, pp. 2044-2061, 2008.

[51] M. Han, X. Gao, J. Z. Su, and S. Nie, "Quantum-dot-tagged microbeads for multiplexed optical coding of biomolecules," Nature Biotechnology, vol. 19, no. 7, pp. 631-635, 2001.

[52] G. Wang, P. Zhang, H. Dou et al., "Efficient incorporation of quantum dots into porous microspheres through a solventevaporation approach," Langmuir, vol. 28, no. 14, pp. 61416150, 2012.

[53] D. Wang, A. L. Rogach, and F. Caruso, "Semiconductor quantum dot-labeled microsphere bioconjugates prepared by stepwise self-assembly," Nano Letters, vol. 2, no. 8, pp. 857-861, 2002.

[54] M. Adamczaka, H. J. Hoelb, G. Gaudernackb, J. Barbasza, K. Szczepanowicza, and P. Warszyńskia, "Polyelectrolyte multilayer capsules with quantum dots for biomedical applications," Colloids and Surfaces B, vol. 90, no. 1, pp. 211-216, 2012.

[55] Z. Zhelev, H. Ohba, and R. Bakalova, "Single quantum dotmicelles coated with silica shell as potentially non-cytotoxic fluorescent cell tracers," Journal of the American Chemical Society, vol. 128, no. 19, pp. 6324-6325, 2006.

[56] P. Zhang, Y. He, Z. Ruan, F. F. Chen, and J. Yang, "Fabrication of quantum dots-encoded microbeads with a simple capillary fluidic device and their application for biomolecule detection," Journal of Colloid and Interface Science, vol. 385, no. 1, pp. 814, 2012.

[57] Q. Yang, Y. Li, T. Song, and J. Chang, "Facile single step preparation of high-performance quantum dot barcodes," Journal of Materials Chemistry, vol. 22, no. 14, pp. 7043-7049, 2012.

[58] J. Lee, Y. Choi, K. Kim et al., "Characterization and cancer cell specific binding properties of anti-EGFR antibody conjugated quantum dots," Bioconjugate Chemistry, vol. 21, no. 5, pp. 940-946, 2010.

[59] T. Pellegrino, L. Manna, S. Kudera et al., "Hydrophobic nanocrystals coated with an amphiphilic polymer shell: a general route to water soluble nanocrystals," Nano Letters, vol. 4, no. 4, pp. 703-707, 2004.

[60] N. Travert-Branger, F. Dubois, O. Carion et al., "Oligomeric PEG-phospholipids for solubilization and stabilization of fluorescent nanocrystals in water," Langmuir, vol. 24, no. 7, pp. 3016-3019, 2008.

[61] J. Liu, X. Yang, K. Wang et al., "Single nanoparticle imaging and characterization of different phospholipid-encapsulated quantum dot micelles," Langmuir, vol. 28, no. 28, pp. 10602 10609, 2012.

[62] N. Travert-Branger, F. Dubois, J. P. Renault et al., "In situ electron-beam polymerization stabilized quantum dot micelles," Langmuir, vol. 27, no. 8, pp. 4358-4361, 2011.

[63] B. Dubertret, P. Skourides, D. J. Norris, V. Noireaux, A. H. Brivanlou, and A. Libchaber, "In vivo imaging of quantum dots encapsulated in phospholipid micelles," Science, vol. 298, no. 5599, pp. 1759-1762, 2002. 
[64] R. Hu, K. T. Yong, I. Roy et al., "Functionalized nearinfrared quantum dots for in vivo tumor vasculature imaging," Nanotechnology, vol. 21, no. 14, Article ID 145105, 2010.

[65] F. Erogbogbo, K. T. Yong, R. Hu et al., "Biocompatible magnetofluorescent probes: luminescent silicon quantum dots coupled with superparamagnetic iron(III) oxide," ACS Nano, vol. 4, no. 9, pp. 5131-5138, 2010.

[66] D. P. Cormode, T. Skajaa, M. M. Van Schooneveld et al., "Nanocrystal core high-density lipoproteins: a multimodality contrast agent platform," Nano Letters, vol. 8, no. 11, pp. 37153723, 2008.

[67] O. Carion, B. Mahler, T. Pons, and B. Dubertret, "Synthesis, encapsulation, purification and coupling of single quantum dots in phospholipid micelles for their use in cellular and in vivo imaging," Nature Protocols, vol. 2, no. 10, pp. 2383-2390, 2007.

[68] R. Koole, M. M. Van Schooneveld, J. Hilhorst et al., "Paramagnetic lipid-coated silica nanoparticles with a fluorescent quantum dot core: a new contrast agent platform for multimodality imaging," Bioconjugate Chemistry, vol. 19, no. 12, pp. 24712479, 2008.

[69] K. T. Yong, "Biophotonics and biotechnology in pancreatic cancer: cyclic RGD-peptide-conjugated type II quantum dots for in vivo imaging," Pancreatology, vol. 10, no. 5, pp. 553-564, 2010.

[70] K. T. Yong, I. Roy, W. C. Law, and R. Hu, "Synthesis of cRGD-peptide conjugated near-infrared CdTe/ZnSe core-shell quantum dots for in vivo cancer targeting and imaging," Chemical Communications, vol. 46, no. 38, pp. 7136-7138, 2010.

[71] V. V. Klimov, M. Ducloy, and V. S. Letokhov, "Strong interaction between a two-level atom and the whispering-gallery modes of a dielectric microsphere: quantum-mechanical consideration," Physical Review A, vol. 59, no. 4, pp. 29963014, 1999.

[72] J. N. Cha, M. H. Bartl, M. S. Wong, A. Popitsch, T. J. Deming, and G. D. Stucky, "Microcavity lasing from block peptide hierarchically assembled quantum dot spherical resonators," Nano Letters, vol. 3, no. 7, pp. 907-911, 2003.

[73] X. Fan, M. C. Lonergan, Y. Zhang, and H. Wang, "Enhanced spontaneous emission from semiconductor nanocrystals embedded in whispering gallery optical microcavities," Physical Review B, vol. 64, no. 11, Article ID 115310, 5 pages, 2001.

[74] W. Sheng, S. Kim, J. Lee, S. W. Kim, K. Jensen, and M. G. Bawendi, "In-situ encapsulation of quantum dots into polymer microspheres," Langmuir, vol. 22, no. 8, pp. 3782-3790, 2006. 

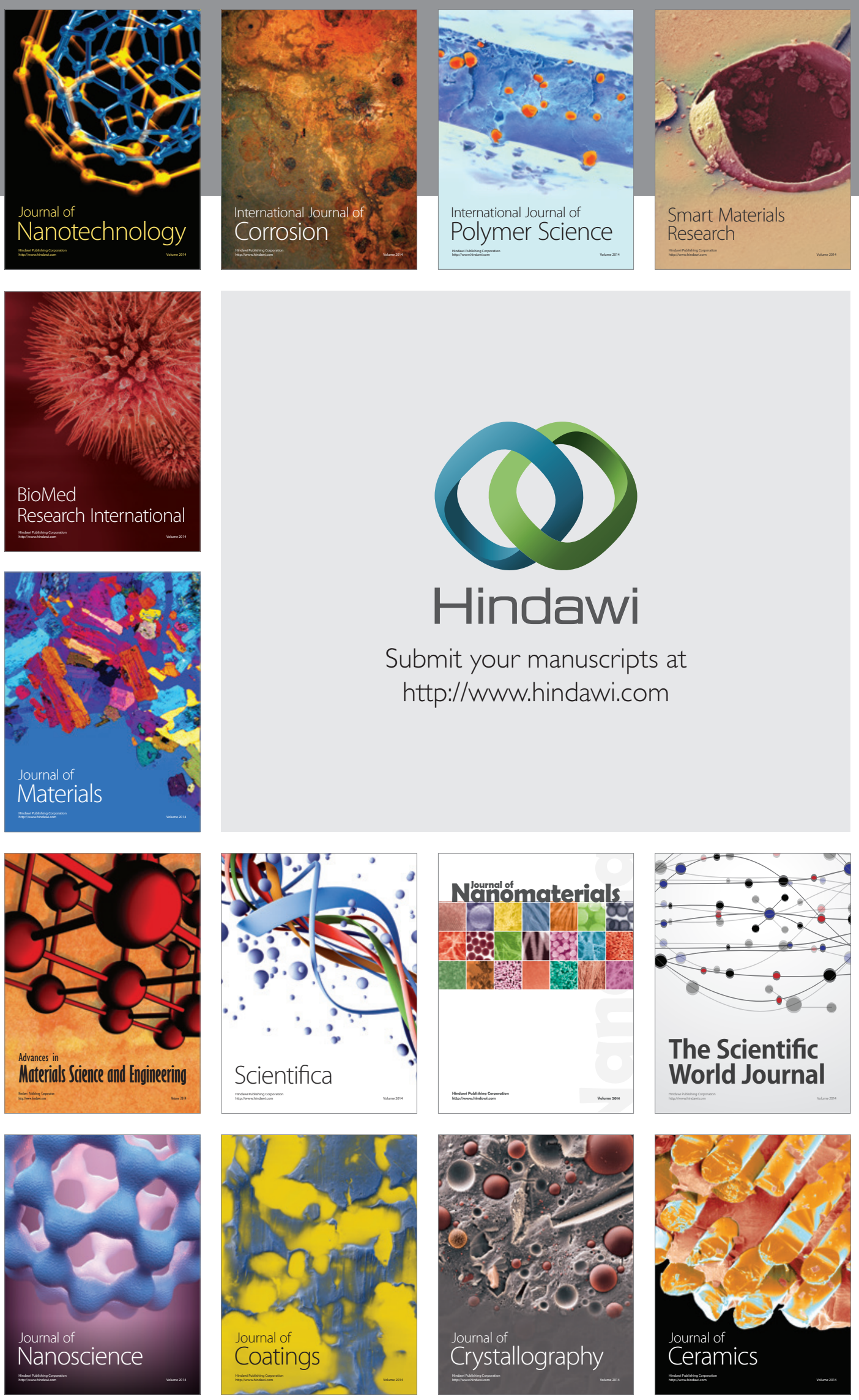

The Scientific World Journal

Submit your manuscripts at

http://www.hindawi.com

\section{World Journal}

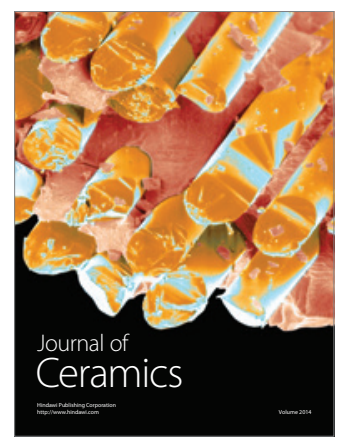

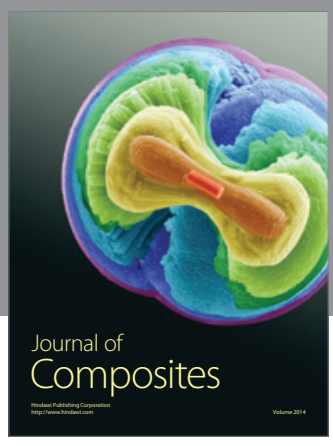
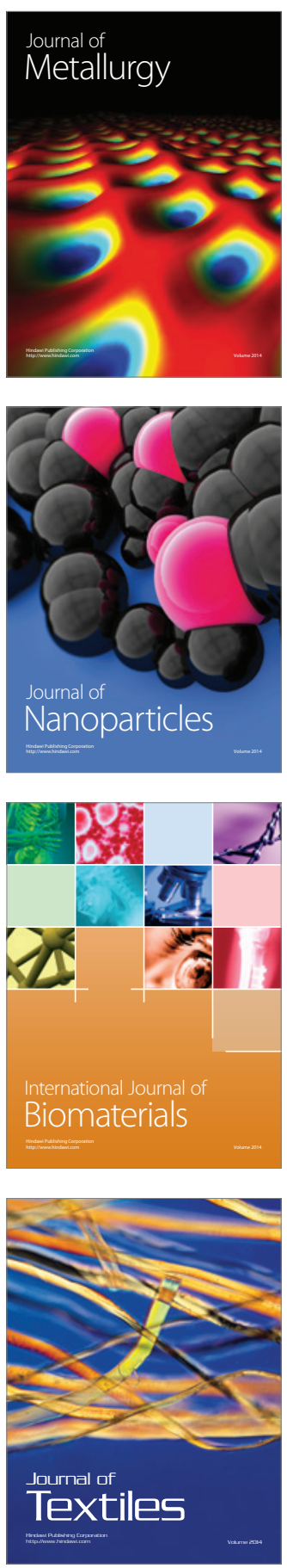